\title{
Minimum-weight codewords as generators of generalized Reed-Muller codes
}

\author{
Peng Ding and Jennifer D. Key*†
}

\begin{abstract}
We establish the range of values of $\rho$, where $0 \leq \rho \leq m(q-1)$, for which the generalized Reed-Muller code $\mathcal{R}_{F_{q}}(\rho, m)$ of length $q^{m}$ over the field $F_{q}$ of order $q$ is spanned by its minimum-weight vectors.
\end{abstract}

Key words: generalized Reed-Muller codes, minimum-weight vectors

\section{Introduction}

The codes of the designs from finite projective and affine geometry are Reed-Muller and generalized Reed-Muller $p$-ary codes, where $p$ is the characteristic of the geometry. The minimum-weight vectors in the codes are the incidence vectors of the blocks of the design, along with scalar multiples, and these generate (i.e. span) the corresponding generalized Reed-Muller code over the prime field, as was shown in work of Delsarte, Goethals and MacWilliams: see [1, Chapter 5] or [2] for full references to this work.

We look here at the generalized Reed-Muller $q$-ary codes for $q$ any prime power, and ask when these are generated by their minimum weight vectors. The work of Delsarte et al. establishes the minimum weight of any of these codes, and the exact nature of the minimum-weight vectors. Delsarte [7, Theorem 10] considers the codes generated by the minimum-weight vectors as extended cyclic codes, and gives the non-roots of the generator polynomial: see also [2, Section 4.3], Charpin [5] or Berger and Charpin [3] for more on the approach to the generalized Reed-Muller codes as extended cyclic codes.

In Section 3 we prove the following:

Theorem 1 Let $C=\mathcal{R}_{F_{q}}(\rho, m)$ be the q-ary generalized Reed-Muller code of order $\rho$ and length $q^{m}$, where $q=p^{t}, p$ is a prime, and $0 \leq \rho \leq m(q-1)$. Then $C$ is generated by its minimum-weight vectors if $m=1$ or $t=1$ or $\rho<p$ or $\rho>(m-1)(q-1)+p^{t-1}-2$. In all other cases it is not generated by its minimum-weight vectors.

\footnotetext{
*Department of Mathematical Sciences, Clemson University, Clemson SC 29634, U.S.A.

${ }^{\dagger}$ Support of NSF grant \#9730992 and ONR grant \#N00014-00-1-0565 acknowledged
} 
We use the method and constructions of Mortimer [9] (see [2, p. 1322]) in our proof. Note also that our statement agrees with the known fact, as shown by Delsarte et al., that in the case when $\rho \equiv 0(\bmod q-1)$, the minimum-weight vectors are constant vectors, and thus only generate a subcode of the same dimension as that of the subfield subcode, which is less than that of the generalized Reed-Muller $q$-ary code if $q$ is not a prime. When $m=1$, the codes are extended Reed-Solomon codes (see [1, Section 5.4]) and hence MDS (maximum-distance-separable) codes, easily seen to be generated by minimum-weight vectors.

We give the necessary definitions, background theory and previous results in Section 2. In Section 3 we build up a proof of the main theorem through a series of lemmas and propositions. In the final section we give some Magma [4] code for computing these dimensions, along with some supporting output.

\section{Terminology and background}

We will use standard terminology for the structures that we need, and in particular we will follow that used in $[1,2]$. These references also contain many related results on the generalized Reed-Muller codes.

Let $q=p^{t}$, where $p$ is a prime, and let $V$ be a vector space of dimension $m$ over the field $F_{q}$ of order $q$. We take $V$ to be the space $F_{q}^{m}$ of $m$-tuples, with standard basis. Our codes will be $q$-ary codes, i.e. codes over $F_{q}$, and the ambient space will be the function space $F_{q}^{V}$, with the usual basis of characteristic functions of the vectors of $V$. We can denote the elements $f$ of $F_{q}^{V}$ by functions of the $m$-variables denoting the coordinates of a variable vector in $V$, i.e. if $\mathbf{x}=\left(x_{1}, x_{2}, \ldots, x_{m}\right) \in V$, then $f \in F_{q}^{V}$ is given by

$$
f=f\left(x_{1}, x_{2}, \ldots, x_{m}\right)
$$

and the $x_{i}$ take values in $F_{q}$. Since every element in $F_{q}$ satisfies $a^{q}=a$, the polynomial functions in the $m$ variables can be reduced modulo $x_{i}^{q}-x_{i}$. Furthermore, every polynomial can be written uniquely as a linear combination of the $q^{m}$ monomial functions

$$
\mathcal{M}=\left\{x_{1}^{i_{1}} x_{2}^{i_{2}} \ldots x_{m}^{i_{m}} \mid 0 \leq i_{k} \leq q-1, k=1,2, \ldots, m\right\} .
$$

For any monomial the degree $\rho$ is the total degree, i.e. $\rho=\sum_{k=1}^{m} i_{k}$ and clearly $0 \leq$ $\rho \leq m(q-1)$.

The generalized Reed-Muller codes can now be defined:

Definition 1 Let $V=F_{q}^{m}$ be the vector space of $m$-tuples, for $m \geq 1$, over the finite field $F_{q}$ of order $q$, where $q=p^{t}$ and $p$ is a prime. For any $\rho$ such that $0 \leq \rho \leq m(q-$ 1 ), the $\rho^{\text {th }}$-order generalized Reed-Muller code $\mathcal{R}_{F_{q}}(\rho, m)$ is the subspace of $F_{q}^{V}$ (with basis the characteristic functions of vectors in $V$ ) of all reduced $m$-variable polynomial 
functions (reduced modulo $x_{i}^{q}-x_{i}$ ) of degree at most $\rho$. Thus

$$
\mathcal{R}_{F_{q}}(\rho, m)=\left\langle x_{1}^{i_{1}} x_{2}^{i_{2}} \cdots x_{m}^{i_{m}} \mid \sum_{k=1}^{m} i_{k} \leq \rho\right\rangle .
$$

These codes are thus codes of length $q^{m}$ and the codewords are obtained by evaluating the $m$-variable polynomials in the subspace at all the points of the vector space $V=F_{q}^{m}$. The Reed-Muller codes are the generalized Reed-Muller codes when $q=2$. Clearly, if $\rho<\nu$ then $\mathcal{R}_{F_{q}}(\rho, m) \subset \mathcal{R}_{F_{q}}(\nu, m)$.

The following result is well known and quoted in [2, Theorem 5.5], for example:

Result 1 For any $\rho$ such that $0 \leq \rho \leq m(q-1)$,

$$
\begin{aligned}
\operatorname{dim}\left(\mathcal{R}_{F_{q}}(\rho, m)\right) & =\sum_{i=0}^{\rho} \sum_{k=0}^{m}(-1)^{k}\left(\begin{array}{c}
m \\
k
\end{array}\right)\left(\begin{array}{c}
i-k q+m-1 \\
i-k q
\end{array}\right) \\
& =\sum_{k=0}^{m}(-1)^{k}\left(\begin{array}{c}
m \\
k
\end{array}\right)\left(\begin{array}{c}
m+\rho-k q \\
\rho-k q
\end{array}\right) .
\end{aligned}
$$

Note: The second formula, simplifying the first, is due to Neil J. Calkin.

Define, for any integers, $k \geq 0$ and $q>1$, the $q$-weight of $k$, written $\operatorname{wt}_{q}(k)$, as $\operatorname{wt}_{q}(k)=\sum_{\nu=0}^{\infty} k_{\nu}$, where $k=\sum_{\nu=0}^{\infty} k_{\nu} q^{\nu}$ is the $q$-ary expansion of $k$. Then an alternative formula for the dimension of $\mathcal{R}_{F_{q}}(\rho, m)$ using the $q$-weight can be given (see $[2])$ : for $0 \leq \rho \leq m(q-1)$,

$$
\operatorname{dim}\left(\mathcal{R}_{F_{q}}(\rho, m)\right)=\mid\left\{u \mid 0 \leq u \leq q^{m}-1 \text { and } \operatorname{wt}_{q}(u) \leq \rho\right\} \mid .
$$

For any code $C$ of length $n$ over a field $F$, an automorphism of $C$ is a permutation $\sigma$ of the $n$ coordinate positions that preserves $C$, i.e. for which, if $c=\left(c_{1}, c_{2}, \ldots, c_{n}\right) \in$ $C$, then $c \sigma \in C$, where $c \sigma$ is defined by $(c \sigma)_{i}=c_{i \sigma^{-1}}$ for $1 \leq i \leq n$. For $0 \leq \rho \leq$ $m(q-1)$, the automorphism group of $\mathcal{R}_{F_{q}}(\rho, m)$ contains the affine general linear group $A G L_{m}\left(F_{q}\right)$ (or $A G L(V)$ ) in its natural action on $V=F_{q}^{m}$ : if $\gamma \in A G L_{m}\left(F_{q}\right)$ is given by

$$
\gamma: \mathbf{v} \mapsto \mathbf{v} A+\mathbf{a},
$$

where $\mathbf{v}, \mathbf{a} \in V=F_{q}^{m}$ and $A$ is a non-singular $m \times m$ matrix over $F_{q}$, then $\mathbf{v} \gamma^{-1}=$ $\mathbf{v} A^{-1}-\mathbf{a} A^{-1}$ and for $f \in \mathcal{R}_{F_{q}}(\rho, m), f \gamma$ is defined by

$$
f \gamma(\mathbf{x})=f\left(\mathbf{x} A^{-1}-\mathbf{a} A^{-1}\right)
$$

where $\mathbf{x}=\left(x_{1}, x_{2}, \ldots, x_{n}\right)$ and the degree in the variables $x_{i}$ is preserved.

The following can be found in [1, Theorem 5.4.2]: 
Result 2 For $\rho<m(q-1)$, the dual code is given by

$$
\mathcal{R}_{F_{q}}(\rho, m)^{\perp}=\mathcal{R}_{F_{q}}(m(q-1)-1-\rho, m) .
$$

In the affine geometry $A G_{m}\left(F_{q}\right)$ defined by $V$ the incidence vectors of the $r$-flats (cosets of dimension $r$ ) can be found in these codes. More generally, if $\rho=r(q-1)+s$, $0 \leq s<q-1$, and for $1 \leq i \leq r, 1 \leq j \leq s, w_{i} \in F_{q}$ are arbitrary and $w_{j}^{\prime} \in F_{q}$ are all distinct, the polynomial

$$
h\left(x_{1}, \ldots, x_{m}\right)=\prod_{i=1}^{r}\left(1-\left(x_{i}-w_{i}\right)^{q-1}\right) \prod_{j=1}^{s}\left(x_{r+1}-w_{j}^{\prime}\right)
$$

has degree $r(q-1)+s=\rho$ and is zero in $V$ unless

$$
\begin{aligned}
x_{i} & =w_{i}, \text { for } i=1, \ldots, r, \\
x_{r+1} & \neq w_{j}^{\prime} \text { for } j=1, \ldots, s .
\end{aligned}
$$

Thus it gives a vector of weight $(q-s) q^{m-r-1}$ which consists of the sum of multiples of incidence vectors of $(q-s)$ parallel $(m-r-1)$-flats all contained in an $(m-r)$-flat in the affine geometry $A G_{m}\left(F_{q}\right)$ : see [1, Theorem 5.5.3]. These are minimum-weight vectors of $\mathcal{R}_{F_{q}}(\rho, m)$. Taking $s=0$ gives the incidence vector of the $(m-r)$-flat defined by the equations $X_{i}=w_{i}$ for $1 \leq i \leq r$.

The following result of Delsarte, Goethals and MacWilliams [6, Theorem 2.6.3] shows that all minimum-weight vectors are of the form given by the polynomial in Equation (5):

Result 3 All the minimum-weight codewords of $\mathcal{R}_{F_{q}}(\rho, m)$, for any values of $m, q$ and $\rho$, can be obtained from the vectors corresponding to polynomial (5) by suitable affine transformations in the affine general linear group $A G L_{m}\left(F_{q}\right)$.

It is well known (see [2]) that the Reed-Muller code $\mathcal{R}_{F_{2}}(r, m)$ is generated by the characteristic vectors of the $(m-r)$-flats in the affine geometry $A G_{m}\left(F_{2}\right)$ and that these are the minimum-weight vectors of $\mathcal{R}_{F_{2}}(r, m)$, of weight is $2^{m-r}$. Thus the Reed-Muller codes are generated by their minimum-weight vectors.

Now we consider the generalized Reed-Muller codes over any finite field $F_{q}$. Mortimer's results [9] are employed extensively in our study and for this we need the following maps:

Definition 2 In the space $F_{q}^{V}$ where $V=F_{q}^{m}$, for $1 \leq i, j \leq m, i \neq j, 0 \leq a_{i} \leq q-1$ and $b$ any integer, define the maps $\delta_{i}^{b}$ and $\varepsilon_{i, j}^{b}$ on the monomials $x_{1}^{a_{1}} x_{2}^{a_{2}} \ldots x_{m}^{a_{m}}$ :

$$
\begin{aligned}
\left(x_{1}^{a_{1}} x_{2}^{a_{2}} \ldots x_{m}^{a_{m}}\right) \delta_{i}^{b} & =\left(\begin{array}{c}
a_{i} \\
b
\end{array}\right) x_{1}^{a_{1}} x_{2}^{a_{2}} \ldots x_{i}^{a_{i}-b} \ldots x_{m}^{a_{m}} \\
\left(x_{1}^{a_{1}} x_{2}^{a_{2}} \ldots x_{m}^{a_{m}}\right) \varepsilon_{i, j}^{b} & =\left(\begin{array}{c}
a_{i} \\
b
\end{array}\right) x_{1}^{a_{1}} x_{2}^{a_{2}} \ldots x_{i}^{a_{i}-b} \ldots x_{j}^{a_{j}+b} \ldots x_{m}^{a_{m}} .
\end{aligned}
$$


The maps are then extended to be linear on the space $F_{q}^{V}$.

Since $\left(\begin{array}{c}a_{i} \\ b\end{array}\right)=0$ for $a_{i}<b, \delta_{i}^{b}$ annihilates the monomial $x_{1}^{a_{1}} x_{2}^{a_{2}} \ldots x_{m}^{a_{m}}$ unless $b \leq a_{i}$; similarly $\varepsilon_{i, j}^{b}$ annihilates $x_{1}^{a_{1}} x_{2}^{a_{2}} \ldots x_{m}^{a_{m}}$ unless $b \leq a_{i}$. Both $\delta_{i}^{0}$ and $\varepsilon_{i, j}^{0}$ are the identity on $\mathcal{M}$ and hence on $F_{q}^{V}$. Notice that if $\delta_{i}^{b}$ does not annihilate a monomial and if $b \neq 0$, then it reduces the degree of the monomial, whereas $\varepsilon_{i, j}^{b}$ keeps the degree fixed or reduces it.

Mortimer [9] (see [2, Lemma 5.32]) proves the following result:

Result 4 The collection of transformations $\varepsilon_{i, j}^{b}$ acts transitively on the set of all monomials of fixed degree (ignoring scalar multiples) when $q=p$ is a prime.

The code $C$ generated by the minimum-weight codewords of $\mathcal{R}_{F_{q}}(\rho, m)$ is invariant under $A G L(V)$. In fact this is true for the code generated by vectors of any fixed given weight in $\mathcal{R}_{F_{q}}(\rho, m)$. We will refer to minimum-weight codewords or vectors as minimum words. We will also use the notation

$$
\left(x_{1}^{a_{1}} x_{2}^{a_{2}} \ldots x_{m}^{a_{m}}\right) f \simeq x_{1}^{b_{1}} x_{2}^{b_{2}} \ldots x_{m}^{b_{m}}
$$

if the monomial on the left is mapped by $f$ to a non-zero scalar multiple of the monomial on the right. We will say that the monomial on the right is obtained from the monomial on the left by the transformation $f$.

The following result due to Mortimer [9] (see [2, Theorem 5.31]) plays an important role in our argument.

Result 5 Let $H$ be a subspace of $F_{q}^{V}$ where $V=F_{q}^{m}, q$ is a prime power and $m \geq 1$. Then $H$ is invariant under the affine general linear group $A G L(V)$ if and only if

- $H$ is invariant under the transformations $\delta_{i}^{b}$ and $\varepsilon_{i, j}^{b}$ for $i \neq j$ and $1 \leq i, j \leq m$ and $0 \leq b \leq q-1$, and

- $H$ is spanned by monomials.

Corollary 2 The code generated by the minimum-weight codewords of $\mathcal{R}_{F_{q}}(\rho, m)$, for any $m \geq 1$, any prime-power $q$ and $0 \leq \rho \leq m(q-1)$, has a monomial basis.

The dimension of the code generated by the minimum-weight vectors follows from Delsarte [7, Theorem 10]:

Result 6 For $m \geq 2, q=p^{t}$ where $p$ is a prime, if $\rho=r(q-1)+s$, where $0 \leq r \leq$ $m-1,0 \leq s<q-1$, then the subcode of $\mathcal{R}_{F_{q}}(\rho, m)$ generated by the minimum-weight codewords has dimension

$$
\mid \bigcup_{0 \leq j \leq s}\left\{z \mid 1 \leq z<q^{m} \text { such that } \operatorname{wt}_{q}\left(p^{k} z\right) \geq(m-r)(q-1)-\left[p^{k} j\right] \text { for } 0 \leq k<t\right\} \mid,
$$

where $[y]$ denotes the residue of $y$ modulo $q-1$. 
When $q=p^{t}$ is not a prime we will need to use $p$-ary expansions: if $n$ and $m$ are integers with $p$-ary expansions $n=\sum_{s=0}^{\infty} n_{s} p^{s}$ and $m=\sum_{s=0}^{\infty} m_{s} p^{s}$, respectively, we will write $(n)_{p} \succeq(m)_{p}$ if $n_{s} \geq m_{s}$ for all $s \geq 0$.

Result 7 (Lucas's Theorem) For $p$ a prime, let $a$ and $b$ be positive integers with p-ary expansions $a=\sum_{s=0}^{v} a_{s} p^{s}$ and $b=\sum_{s=0}^{v} b_{s} p^{s}$. Then

$$
\left(\begin{array}{l}
a \\
b
\end{array}\right) \equiv \prod_{s=0}^{v}\left(\begin{array}{l}
a_{s} \\
b_{s}
\end{array}\right)(\bmod p) .
$$

In the proofs in the next section we will need the following definition:

Definition 3 Given a monomial $x_{1}^{a_{1}} x_{2}^{a_{2}} \ldots x_{m}^{a_{m}}$, where $0 \leq a_{i} \leq q-1$ for $1 \leq i \leq m$ and $q=p^{t}$, suppose that the $p$-ary expansion of $a_{i}$ is $a_{i}=\sum_{j=0}^{t-1} a_{i, j} p^{j}$, where $0 \leq a_{i, j} \leq$ $p-1$. For $0 \leq k \leq t-1$, the $k$-component-degree of $x_{1}^{a_{1}} x_{2}^{a_{2}} \ldots x_{m}^{a_{m}}$, denoted by $\mathrm{cdeg}_{k}$, is defined by

$$
\operatorname{cdeg}_{k}\left(x_{1}^{a_{1}} x_{2}^{a_{2}} \ldots x_{m}^{a_{m}}\right)=\sum_{i=1}^{m} a_{i, k} .
$$

Given any integer $b$ with $p$-ary expansion $b=\sum_{i=0}^{\infty} b_{i} p^{i}$, where $0 \leq b_{i} \leq p-1$, we define

$$
\bar{b}=\min \left\{i \mid b_{i} \neq 0\right\} \text {. }
$$

\section{Minimum-weight words as generators}

In this section we will prove our main theorem through a series of lemmas and propositions.

Proposition 3 For any $\rho, m$ and $q=p$ a prime, $\mathcal{R}_{F_{p}}(\rho, m)$ is generated by its minimum-weight codewords.

Proof: Suppose $\rho=r(p-1)+s$, where $0 \leq s<p-1$, and let $C$ be the code generated by the minimum words of $\mathcal{R}_{F_{p}}(\rho, m)$. Then $C$ is clearly invariant under $A G L(V)$ and so Result 5 applies. It can be seen that the monomial $x_{1}^{p-1} x_{2}^{p-1} \ldots x_{r}^{p-1} x_{r+1}^{s^{\prime}}$, for any $s^{\prime} \leq s$, is one term in the polynomial function of Equation (5) that gives a minimum word. From Result 5, it must be a monomial in the monomial basis of $C$. All the monomials transformed from it by some transformation of type $\delta_{i}^{b}$ are in $C$ as well. For any $a$ such that $0 \leq a \leq p-1$, we have $\left(\begin{array}{c}p-1 \\ a\end{array}\right) \neq 0$, and hence, given a monomial $x_{1}^{i_{1}} x_{2}^{i_{2}} \ldots x_{r}^{i_{r}} x_{r+1}^{s^{\prime}}$ where $0 \leq i_{1}, i_{2}, \ldots, i_{r} \leq p-1,0 \leq s^{\prime} \leq s$, we have

$$
\left(x_{1}^{p-1} x_{2}^{p-1} \ldots x_{r}^{p-1} x_{r+1}^{s}\right) \delta_{r+1}^{s-s^{\prime}} \delta_{r}^{p-1-i_{r}} \ldots \delta_{1}^{p-1-i_{1}} \simeq x_{1}^{i_{1}} x_{2}^{i_{2}} \ldots x_{r}^{i_{r}} x_{r+1}^{s^{\prime}} .
$$


Therefore $C$ must contain the monomial $x_{1}^{i_{1}} x_{2}^{i_{2}} \ldots x_{r}^{i_{r}} x_{r+1}^{s^{\prime}}$. Hence $C$ contains monomials with degrees ranging from 0 to $\rho$. It follows from Result 4 that $C$ contains all the monomial of degree up to $\rho$. Hence $C$ is precisely $\mathcal{R}_{F_{p}}(\rho, m)$ by Definition 1 .

Note: This can also be deduced, in this case where $q=p$ is a prime, from Delsarte's result for the formula of the dimension of the code spanned by the minimum words: see Equation (2) and Result 6.

Now we consider the generalized Reed Muller code $\mathcal{R}_{F_{q}}(\rho, m)$ where $q=p^{t}$ is not a prime. Then $q-1=\sum_{i=0}^{t-1}(p-1) p^{i}$ and thus $(q-1)_{p} \succeq(a)_{p}$ for $0 \leq a \leq q-1$. It follows from Lucas's theorem that $\left(\begin{array}{c}q-1 \\ a\end{array}\right) \not \equiv 0(\bmod p)$. In $\mathcal{R}_{F_{q}}(\rho, m)$, where $\rho=r(q-1)+s$, $x_{1}^{q-1} x_{2}^{q-1} \ldots x_{r}^{q-1} x_{r+1}^{s}$ is one term in the polynomial of Equation (5). Using the same argument as in the proof of Proposition 3, we can conclude that the monomials of type

$$
x_{1}^{i_{1}} x_{2}^{i_{2}} \ldots x_{r}^{i_{r}} x_{r+1}^{s^{\prime}}, 0 \leq i_{1}, i_{2}, \ldots, i_{r} \leq q-1,0 \leq s^{\prime} \leq s,
$$

are in the monomial basis of the code $C$ generated by the minimum words of $\mathcal{R}_{F_{q}}(\rho, m)$.

Lemma 4 Let $\mathcal{B}$ be the monomial basis of the code $C$ generated by minimum words of $\mathcal{R}_{F_{q}}(\rho, m)$. Any monomial in $\mathcal{B}$ can be obtained from some monomial of type (10) having the same or greater degree, by some transformations of type $\varepsilon_{i, j}^{b}$.

Proof: Since $C$ is invariant under $A G L_{m}\left(F_{q}\right)$, it follows from Results 3 and 5 that any monomial $M(\mathbf{x})$ in $\mathcal{B}$ must be a monomial term in a polynomial that is mapped from the polynomial $h(\mathbf{x})$ of Equation (5) by some transformation in $A G L_{m}\left(F_{q}\right)$. Any element of $A G L_{m}\left(F_{q}\right)$ is the product of a translation and a linear transformation.

We consider the following translation for any fixed $i$ and $u \in F_{q}$ :

$$
\sigma_{i}^{u}:\left(x_{1}, x_{2}, \ldots, x_{m}\right) \longmapsto\left(x_{1}, x_{2}, \ldots, x_{i-1}, x_{i}-u, x_{i+1}, \ldots, x_{m}\right) .
$$

The polynomial of Equation (5) can be written as $h\left(x_{1}, x_{2}, \ldots, x_{m}\right)=\sum_{j} h_{j} x_{i}^{j}$ where the $h_{j}$ are polynomials independent of $x_{i}$. Then it follows that $h \sigma_{i}^{u}(\mathbf{x})$ (see notation in Equation 4) satisfies the following:

$$
\begin{aligned}
h \sigma_{i}^{u}(\mathbf{x}) & =\sum_{j} h_{j}\left(x_{i}+u\right)^{j}=\sum_{j} h_{j} \sum_{b}\left(\begin{array}{l}
j \\
b
\end{array}\right) x_{i}^{j-b} u^{b} \\
& =\sum_{b} u^{b} \sum_{j}\left(\begin{array}{l}
j \\
b
\end{array}\right) h_{j} x_{i}^{j-b}=\sum_{b} u^{b}(h(\mathbf{x})) \delta_{i}^{b} .
\end{aligned}
$$

Obviously the monomials in $(h(\mathbf{x})) \delta_{i}^{b}$ are of type (10) and thus the translations map $h(\mathbf{x})$ to polynomials that contain only monomial terms of type (10).

Now for any fixed $i, j, i \neq j$, consider the transvection $\lambda_{i, j}$ where

$$
\lambda_{i, j}:\left(x_{1}, x_{2}, \ldots, x_{m}\right) \longmapsto\left(x_{1}, x_{2}, \ldots, x_{i}-x_{j}, \ldots, x_{m}\right),
$$


i.e. $\left(x_{1}, \ldots, x_{m}\right) \lambda_{i, j}=\left(y_{1}, \ldots, y_{m}\right)$ where $y_{k}=x_{k}$ for $k \neq i$ and $y_{i}=x_{i}-x_{j}$. For a polynomial $f$ we write $f\left(x_{1}, \ldots, x_{m}\right)=\sum_{r, s} f_{r, s} x_{i}^{r} x_{j}^{s}$, where the $f_{r, s}$ are polynomials independent of $x_{i}$ and $x_{j}$. Then it follows directly that

$$
\begin{aligned}
f \lambda_{i, j}(\mathbf{x}) & =\sum_{r, s} f_{r, s}\left(x_{i}+x_{j}\right)^{r} x_{j}^{s}=\sum_{r, s} f_{r, s} \sum_{b}\left(\begin{array}{l}
r \\
b
\end{array}\right) x_{i}^{r-b} x_{j}^{s+b} \\
& =\sum_{b} \sum_{r, s}\left(\begin{array}{l}
r \\
b
\end{array}\right) f_{r, s} x_{i}^{r-b} x_{j}^{s+b}=\sum_{b}(f(\mathbf{x})) \varepsilon_{i, j}^{b} .
\end{aligned}
$$

Thus the monomials that can be obtained from $h(\mathbf{x})$ by translations or transvections are those that can be obtained from the monomials in $h(\mathbf{x})$ by the maps $\delta_{i}^{b}$ and the $\varepsilon_{i, j}^{b}$. For any nonsingular matrix $A$, ignoring scalar products, $A$ can be written as the product of matrices of the transformations (12). Thus it can be seen from the above procedures that any monomial $M(\mathbf{x})$ in $\mathcal{B}$ is obtained from some monomial of type (10) of no smaller degree by some transformations of type $\varepsilon_{i, j}^{b}$.

Example 1 In the code $\mathcal{R}_{F_{4}}(3,2)$, the incidence polynomial (5) is $h\left(x_{1}, x_{2}\right)=1$ $\left(x_{1}-\alpha\right)^{3}$ for some $\alpha \in F_{4}$. Here $m=2, r=1$ and $s=0$. According to Lemma 4, all the monomials of degree 2 in the code generated by minimum-weight codewords must be obtained from $x_{1}^{2}$ or $x_{1}^{3}$ by transformations of type (7). It is easy to verify that the monomial $x_{1} x_{2} \in \mathcal{R}_{F_{4}}(3,2)$ cannot be obtained in this way, since, for example, $x_{1}^{2} \varepsilon_{1,2}^{1}=\left(\begin{array}{l}2 \\ 1\end{array}\right) x_{1} x_{2}=0$. Thus $\mathcal{R}_{F_{4}}(3,2)$ is not generated by its minimum-weight vectors. Note that the binary subfield subcode of $\mathcal{R}_{F_{4}}(3,2)$ is generated by its minimum-weight vectors, and a basis of nine vectors is given in [1, Example 5.7.1, page 187]. These involve the nine monomials $\left\{1, x_{1}, x_{1}^{2}, x_{1}^{3}, x_{2}, x_{2}^{2}, x_{2}^{3}, x_{1} x_{2}^{2}, x_{1}^{2} x_{2}\right\}$, which form a basis for the code generated by the minimum-weight vectors over $F_{4}$.

Lemma 5 Given two monomials $M_{A}=x_{1}^{a_{1}} x_{2}^{a_{2}} \ldots x_{m}^{a_{m}}$ and $M_{B}=x_{1}^{b_{1}} x_{2}^{b_{2}} \ldots x_{m}^{b_{m}}$, if $\operatorname{cdeg}_{k}\left(M_{A}\right)=\operatorname{cdeg}_{k}\left(M_{B}\right)$ for $0 \leq k \leq t-1$ then $M_{B}$ can be obtained from $M_{A}$ by transformations of type $\varepsilon_{i, j}^{b}$.

Proof: (Refer to Definition 3 for the notation here.) Clearly $\operatorname{deg}\left(M_{A}\right)=\operatorname{deg}\left(M_{B}\right)$ since $\operatorname{cdeg}_{k}\left(M_{A}\right)=\operatorname{cdeg}_{k}\left(M_{B}\right)$ for $0 \leq k \leq t-1$. As before, letting $a_{i, k}$ and $b_{i, k}$ be the $k$-th $p$-ary digits of $a_{i}$ and $b_{i}$ respectively, we have $\sum_{l=1}^{m} a_{l, k}=\sum_{l=1}^{m} b_{l, k}$ for $0 \leq k \leq t-1$. Fix $k$ in the range $0 \leq k \leq t-1$. The proof will follow if we can prove that $a_{l, k}$ can be changed to $b_{l, k}$ by transformations of the type $\varepsilon_{i, j}^{b}$ for $1 \leq l \leq m$. Without loss of generality, assume that

$$
a_{1, k} \geq b_{1, k}, a_{2, k} \geq b_{2, k}, \ldots, a_{d, k} \geq b_{d, k},
$$

and

$$
a_{d+1, k}<b_{d+1, k}, a_{d+2, k}<b_{d+2, k}, \ldots, a_{m, k}<b_{m, k} .
$$


Then

$$
\sum_{i=1}^{d}\left(a_{i, k}-b_{i, k}\right)=\sum_{i=1}^{m-d}\left(b_{d+i, k}-a_{d+i, k}\right) .
$$

For $0<i<d+1$ and $a_{i, k}>b_{i, k}$, there exists $j$ such that $d<j \leq m$ and $a_{j, k}<b_{j, k}$, and

$$
\left(M_{A}\right) \varepsilon_{i, j}^{p^{k}}=\left(\begin{array}{c}
a_{i} \\
p^{k}
\end{array}\right) x_{1}^{a_{1}} x_{2}^{a_{2}} \ldots x_{i}^{\left(a_{i}-p^{k}\right)} \ldots x_{j}^{\left(a_{j}+p^{k}\right)} \ldots x_{m}^{a_{m}} \neq 0
$$

by Lucas's theorem, and reduces $a_{i, k}$ by 1 and increases $a_{j, k}$ by 1 . If this procedure is applied repeatedly, then $a_{i, k}$ can be reduced to $b_{i, k}$. If we reduce all the $a_{i, k}$ to $b_{i, k}$ for all $0<i<d+1$, then simultaneously $a_{j, k}$ is increased to $b_{j, k}$ for all $d<j \leq m$ by the above procedure. This can be done for each value of $k$.

Example 2 For $q=2^{4}, m=4$, let $A_{k}$ and $B_{k}$ be the $k$-component-degrees of $M_{A}=$ $x_{1}^{3} x_{2}^{5} x_{3}^{2} x_{4}^{8}$ and $M_{B}=x_{1}^{7} x_{2} x_{3}^{10}$ respectively, for $0 \leq k \leq 3$. It is easy to check that $A_{k}=B_{k}$ for all $k$ and $A_{0}=2, A_{1}=2, A_{2}=1, A_{3}=1$. It follows from Lemma 5 that there exist transformations of type $\varepsilon_{i, j}^{b}$ such that $x_{1}^{7} x_{2} x_{3}^{10}$ can be obtained from $x_{1}^{3} x_{2}^{5} x_{3}^{2} x_{4}^{8}$ by these transformations. For instance, $\left(x_{1}^{3} x_{2}^{5} x_{3}^{2} x_{4}^{8}\right) \varepsilon_{2,1}^{4} \varepsilon_{4,3}^{8} \simeq x_{1}^{7} x_{2} x_{3}^{10}$.

Lemma 6 Let $M=x_{1}^{a_{1}} x_{2}^{a_{2}} \ldots x_{m}^{a_{m}}$ and let $A_{k}$ denote the $k$-component degree of $M$. For any integer $b$ and any $k$ such that $0 \leq k \leq t-1$, if $k \leq \bar{b}$ then $\operatorname{cdeg}_{k}\left(M \varepsilon_{i, j}^{b}\right) \leq A_{k}$ for $1 \leq i, j \leq m$.

Proof: (Recall that $\bar{b}$ is defined in Equation (9).) Fix $i, j$ in the range. If $M \varepsilon_{i, j}^{b}=0$, then the claim is trivially true. If $M \varepsilon_{i, j}^{b} \neq 0$, then $a_{i} \succeq b$. If $k<\bar{b}$, the $k$-th digit of $a_{i}-b$ is the same as that of $a_{i}$, and the $k$-th digit of $a_{j}+b$ is the same as that of $a_{j}$. If $k=\bar{b}$, the $k$-th digit of $a_{i}-b$ is less than that of $a_{i}$ by $b_{k}$, and the $k$-th digit of $a_{j}+b$ is greater than that of $a_{j}$ by at most $b_{k}$. This gives the stated result.

Lemma 7 Let $M_{A}=x_{1}^{a_{1}} x_{2}^{a_{2}} \ldots x_{m}^{a_{m}}$ and $M_{B}=x_{1}^{b_{1}} x_{2}^{b_{2}} \ldots x_{m}^{b_{m}}$ be two monomials and let $A_{k}, B_{k}$ be their $k$-component-degrees respectively, for $0 \leq k \leq t-1$. Suppose that $A_{k}=B_{k}$, for $0 \leq k \leq l-1$ and $A_{l}<B_{l}$ for some $l \leq t-1$. Then $M_{B}$ cannot be obtained from $M_{A}$ by transformations of the type $\varepsilon_{i, j}^{b}$.

Proof: We use induction on $l$. If $l=0$, then $A_{0}<B_{0}$. From Lemma $6, A_{0}$ cannot be increased by any transformations $\varepsilon_{i, j}^{b}$ since $0 \leq \bar{b}$.

Assume that the result holds for $0 \leq l \leq d-1$ and suppose $A_{k}=B_{k}$ for $0 \leq k \leq d-1$, and $A_{d}<B_{d}$. If $M_{B}$ can be obtained from $M_{A}$, then $A_{d}$ can be increased to $B_{d}$ by some transformations of the type $\varepsilon_{i, j}^{c}$. Thus for some $c$ and $i, j, M_{C}=M_{A} \varepsilon_{i, j}^{c}$ has $d$-component degree $C_{d}$ where $B_{d} \geq C_{d}>A_{d}$, and $M_{B}$ can be obtained from $M_{C}$ by further transformations. It follows from Lemma 6 that $d>\bar{c}$ and for some $l$ such that 
$\bar{c} \leq l \leq d-1, C_{l}<A_{l}=B_{l}$, and for $k<l, C_{k}=A_{k}=B_{k}$. By the induction hypothesis, $M_{B}$ cannot be obtained from $M_{C}$, contradicting our assumption. This completes the proof.

The generalized Reed-Muller code $\mathcal{R}_{F_{q}}(\rho, 1)$ is an extended Reed-Solomon code and is an MDS code; thus it is clearly generated by its minimum-weight codewords. Thus from now on we can assume that $m>1$.

Proposition 8 For any $m$ and $q=p^{t}$, if $\rho<p$ then $\mathcal{R}_{F_{q}}(\rho, m)$ is generated by its minimum-weight codewords.

Proof: Since $\rho<p<q$, the polynomial of Equation (5) is $\prod_{j=1}^{\rho}\left(x_{1}-w_{j}^{\prime}\right)$. It follows from Lemma 4 that all the monomials in the code generated by the minimum words are the monomials which can be obtained from $1, x_{1}, x_{1}^{2}, \ldots, x_{1}^{\rho}$ by transformations of type $\varepsilon_{i, j}^{b}$. Since $\left(\begin{array}{l}a \\ b\end{array}\right) \neq 0$ if $a>b$ and $a<p, b<p$, all the monomials with degree no greater than $\rho$ can be obtained by transformations of type $\varepsilon_{i, j}^{b}$.

Proposition 9 For $m \geq 2$, and $q=p^{t}$, if $p \leq \rho<q$ then $\mathcal{R}_{F_{q}}(\rho, m)$ is not generated by its minimum-weight codewords.

Proof: Since $\rho \leq q-1$, the polynomial of Equation (5) is $\prod_{j=1}^{\rho}\left(x_{1}-w_{j}^{\prime}\right)$ if $\rho<q-1$, or $1-\left(x_{1}-w_{1}\right)^{q-1}$ if $\rho=q-1$. The type (10) monomials are $x_{1}^{r}$ for $0 \leq r \leq \rho$. For the monomial $x_{1}^{p-1} x_{2}, \operatorname{cdeg}_{0}\left(x_{1}^{p-1} x_{2}\right)=p$ which is greater than $\operatorname{cdeg}_{0}\left(x_{1}^{r}\right)$ for $r \leq q-1$. It follows from Lemma 6 that $x_{1}^{p-1} x_{2}$ cannot be obtained from $x_{1}^{r}$ for any $r \leq q-1$ by transformations of the type $\varepsilon_{i, j}^{b}$. According to Lemma 4 , the monomial $x_{1}^{p-1} x_{2}$ is not in the code generated by the minimum-weight codewords in $\mathcal{R}_{F_{q}}(\rho, m)$.

Proposition 10 For $m \geq 3$, if $\rho=r(q-1)+s$, where $0<r \leq m-2$ and $0 \leq s \leq q-2$, then $\mathcal{R}_{F_{q}}(\rho, m)$ is not generated by its minimum-weight codewords.

Proof: Since $0<r \leq m-2$ and $2(p-1) \leq p^{2}-p, x_{1}^{p-1} x_{2}^{p-1} \ldots x_{r+1}^{p-1} x_{r+2}^{p-1}$ is a monomial in $\mathcal{R}_{F_{q}}(\rho, m)$ and its 0 -component-degree is $(r+2)(p-1)$. According to Lemma $7, x_{1}^{p-1} x_{2}^{p-1} \ldots x_{r+1}^{p-1} x_{r+2}^{p-1}$ cannot be transformed from monomials of type (10) by transformations $\varepsilon_{i, j}^{b}$, since $x_{1}^{i_{1}} x_{2}^{i_{2}} \ldots x_{r}^{i_{r}} x_{r+1}^{s}$ has 0 -component-degree strictly less than $(r+2)(p-1)$ for any $i_{1}, i_{2}, \ldots, i_{r}, s$. Therefore $x_{1}^{p-1} x_{2}^{p-1} \ldots x_{r+1}^{p-1} x_{r+2}^{p-1}$ is not in the code generated by the minimum-weight codewords in $\mathcal{R}_{F_{q}}(\rho, m)$ by Lemma 4 .

Proposition 11 If $m \geq 2, q=p^{t}$ and $\rho=(m-1)(q-1)+s$ where $0 \leq s<p^{t-1}-1$ then $\mathcal{R}_{F_{q}}(\rho, m)$ is not generated by its minimum-weight codewords. 
Proof: Let $r=p^{t-1}-1=\sum_{i=0}^{t-2}(p-1) p^{i}$. The monomial $x_{1}^{r} x_{2}^{r} \ldots x_{m}^{r}$ is in $\mathcal{R}_{F_{q}}(\rho, m)$ and has the maximum $k$-component-degree where $0 \leq k \leq t-2$. Since $r>s$, the $(t-2)$-component-degree of $x_{1}^{r} x_{2}^{r} \ldots x_{m}^{r}$ must be greater than that of any monomial of type (10) in the code and hence it cannot be obtained from type (10) monomials by transformations of type $\varepsilon_{i, j}^{b}$, by Lemma 7 . Thus the monomial $x_{1}^{r} x_{2}^{r} \ldots x_{m}^{r}$ is not in the code generated by the minimum-weight codewords in $\mathcal{R}_{F_{q}}(\rho, m)$.

Proposition 12 For $m \geq 2$ and $q=p^{t}$, if $\rho=(m-1)(q-1)+s$ where $s \geq p^{t-1}-1$, then $\mathcal{R}_{F_{q}}(\rho, m)$ is generated by its minimum-weight codewords.

Proof: For any monomial $M=x_{1}^{d_{1}} x_{2}^{d_{2}} \ldots x_{m}^{d_{m}}$ with degree no greater than $\rho$, if we can show that it can be obtained from some monomial of type (10) with the same or greater degree less than $\rho$ by transformations of type $\varepsilon_{i, j}^{b}$, then the stated result is proved. If $d_{m}=0$ this is clear.

Suppose that $\operatorname{deg}(M)=(m-1)(q-1)+s^{\prime}$ where $0 \leq s^{\prime} \leq s$. The monomial $x_{1}^{q-1} x_{2}^{q-1} \ldots x_{m-1}^{q-1} x_{m}^{s^{\prime}}$ is of type (10). Since $\left(\begin{array}{c}q-1 \\ a\end{array}\right) \neq 0$ for $0 \leq a \leq q-1$, it follows that

$$
\left(x_{1}^{q-1} x_{2}^{q-1} \ldots x_{m-1}^{q-1} x_{m}^{s^{\prime}}\right) \varepsilon_{1, m}^{q-1-d_{1}} \varepsilon_{2, m}^{q-1-d_{2}} \ldots \varepsilon_{m-1, m}^{q-1-d_{m-1}} \simeq M .
$$

If $\operatorname{deg}(M)<(m-1)(q-1)$ let $M_{t}^{\prime}=x_{1}^{d_{1}} x_{2}^{d_{2}} \ldots x_{m-1}^{d_{m-1}}$ and write

$$
d_{m}=\operatorname{deg}(M)-\operatorname{deg}\left(M_{t}^{\prime}\right)=f_{t-1} p^{t-1}+r_{t-1}, \quad 0 \leq r_{t-1} \leq p^{t-1}-1 \leq s .
$$

If $\operatorname{cdeg}_{t-1}\left(M_{t}^{\prime}\right)+f_{t-1} \leq(m-1)(p-1)$, we construct a monomial $M_{t-1}^{\prime}=x_{1}^{d_{1}^{\prime}} x_{2}^{d_{2}^{\prime}} \ldots x_{m-1}^{d_{m-1}^{\prime}}$ from $M_{t}^{\prime}$ by adding $f_{t-1} p^{t-1}$ to the degree of $M_{t}^{\prime}$ such that $\operatorname{cdeg}_{t-1}\left(M_{t-1}^{\prime}\right)=f_{t-1}+$ $\operatorname{cdeg}_{t-1}\left(M_{t}^{\prime}\right)$, and $\operatorname{cdeg}_{i}\left(M_{t-1}^{\prime}\right)=\operatorname{cdeg}_{i}\left(M_{t}^{\prime}\right)$ for $0 \leq i \leq t-2$. Since $r_{t-1} \leq s$, the monomial $M_{t-1}^{\prime} x_{m}^{r_{t-1}}$ is of type (10) and clearly $d_{j}^{\prime} \succeq d_{j}^{\prime}-d_{j}$ for $1 \leq j \leq m-1$. Thus

$$
\left(M_{t-1}^{\prime} x_{m}^{r_{t-1}}\right) \varepsilon_{1, m}^{d_{1}^{\prime}-d_{1}} \varepsilon_{2, m}^{d_{2}^{\prime}-d_{2}} \ldots \varepsilon_{m-1, m}^{d_{m-1}^{\prime}-d_{m-1}} \simeq M
$$

and the proof is complete.

If $\operatorname{cdeg}_{t-1}\left(M_{t}^{\prime}\right)+f_{t-1}>(m-1)(p-1)$, we construct $M_{t-1}^{\prime}=x_{1}^{d_{1}^{\prime}} x_{2}^{d_{2}^{\prime}} \ldots x_{m-1}^{d_{m-1}^{\prime}}$ by adding $\left((m-1)(p-1)-\operatorname{cdeg}_{t-1}\left(M_{t}^{\prime}\right)\right) p^{t-1}$ to the degree of $M_{t}^{\prime}$ such that $\operatorname{cdeg}_{t-1}\left(M_{t-1}^{\prime}\right)=$ $(m-1)(p-1)$, and $\operatorname{cdeg}_{i}\left(M_{t-1}^{\prime}\right)=\operatorname{cdeg}_{i}\left(M_{t}^{\prime}\right)$ for $0 \leq i \leq t-2$. Then write

$$
\operatorname{deg}(M)-\operatorname{deg}\left(M_{t-1}^{\prime}\right)=f_{t-2} p^{t-2}+r_{t-2}, \quad 0 \leq r_{t-2} \leq p^{t-2}-1 \leq s .
$$

Now we construct $M_{t-2}^{\prime}$ from $M_{t-1}^{\prime}$ by the same procedure we used to construct $M_{t-1}^{\prime}$ from $M_{t}^{\prime}$, except that we now only increase $\operatorname{cdeg}_{t-2}\left(M_{t-1}^{\prime}\right)$ : using the same argument as above we see that if $\operatorname{cdeg}_{t-2}\left(M_{t-1}^{\prime}\right)+f_{t-2} \leq(m-1)(p-1)$, then the proof is done, and if $\operatorname{cdeg}_{t-2}\left(M_{t-1}^{\prime}\right)+f_{t-2}>(m-1)(p-1)$, we need to construct $M_{t-3}^{\prime}$. Because $\operatorname{deg}(M) \leq(m-1)(q-1)$, the recursive construction will terminate and produce the monomial we need.

The following example illustrates the algorithm we use in proving Proposition 12. 
Example 3 In $\mathcal{R}_{F_{27}}(86,4)$ we have $m=4, r=t=3, s=3^{2}-1,(m-1)(q-1)=78$ and $(m-1)(p-1)=6$. A monomial of type (10) has the form $x_{1}^{i_{1}} x_{2}^{i_{2}} x_{3}^{i_{3}} x_{4}^{i_{4}}$, where $0 \leq i_{1}, i_{2}, i_{3} \leq 26$, and $0 \leq i_{4} \leq 8$. The monomial $M=x_{1}^{10} x_{2}^{25} x_{3}^{25} x_{4}^{25}$ is not of type (10); here $\operatorname{deg}(M)=85=3 \times 26+7>78$, so Proposition 12 yields

$$
\left(x_{1}^{26} x_{2}^{26} x_{3}^{26} x_{4}^{7}\right) \varepsilon_{1,4}^{16} \varepsilon_{2,4}^{1} \varepsilon_{3,4}^{1} \simeq M .
$$

Now consider $M=x_{1}^{12} x_{2}^{18} x_{3}^{21} x_{4}^{26}$ of degree $77=2 \times 26+25<78$. Then $M_{3}^{\prime}=x_{1}^{12} x_{2}^{18} x_{3}^{21}$ and

$$
\operatorname{deg}(M)-\operatorname{deg}\left(M_{3}^{\prime}\right)=26=2 \times 9+8, \quad \operatorname{cdeg}_{2}\left(M_{3}^{\prime}\right)+2=7>6 .
$$

Thus we add $1 \times 9$ to the degree to obtain $M_{2}^{\prime}=x_{1}^{21} x_{2}^{18} x_{3}^{21}$ and

$$
\operatorname{deg}(M)-\operatorname{deg}\left(M_{2}^{\prime}\right)=17=5 \times 3+2, \quad \operatorname{cdeg}_{1}\left(M_{2}^{\prime}\right)+5=7>6 .
$$

Thus we add $4 \times 3$ to the degree to obtain $M_{1}^{\prime}=x_{1}^{24} x_{2}^{24} x_{3}^{24}$ and

$$
\operatorname{deg}(M)-\operatorname{deg}\left(M_{1}^{\prime}\right)=5 \times 1+0, \quad \operatorname{cdeg}_{0}\left(M_{1}^{\prime}\right)+5=5<6 .
$$

So finally we add $5 \times 1$ to the degree to obtain $M_{0}^{\prime}=x_{1}^{26} x_{2}^{26} x_{3}^{25}$, and obtain

$$
\left(M_{0}^{\prime} x_{4}^{0}\right) \varepsilon_{1,4}^{14} \varepsilon_{2,4}^{8} \varepsilon_{3,4}^{4} \simeq M .
$$

The propositions of this section, along with the observation that $\mathcal{R}_{F_{q}}(m(q-1), m)$ is trivially seen to be generated by its minimum-weight codewords, it being the full space, completes the proof of Theorem 1 .

\section{Note:}

1. If $\rho=r(q-1)$ then the minimum-weight vectors of $\mathcal{R}_{F_{q}}(\rho, m)$ are the incidence vectors of the $(m-r)$-flats, and the code generated by them is a subfield subcode: see $\left[1\right.$, Chapter 5]. This is only equal to $\mathcal{R}_{F_{q}}(\rho, m)$ if $q$ is a prime. Our theorem agrees with this.

2. The dual code of $\mathcal{R}_{F_{q}}(\rho, m)$ may be generated by its minimum words while $\mathcal{R}_{F_{q}}(\rho, m)$ is not. For example, $\mathcal{R}_{F_{9}}(12,2)$ is generated by its minimum words, but $\mathcal{R}_{F_{9}}(12,2)^{\perp}=$ $\mathcal{R}_{F_{9}}(3,2)$ is not. In fact if $m>1$ and $q$ is not prime, only in the case $q=4$ will $\mathcal{R}_{F_{q}}(\rho, m)$ be generated by its minimum words if and only if its dual is generated by its minimum words.

3. Actual bases of minimum-weight vectors are known only in some specific cases: see Gao and Key [8] for a discussion, and where the case $\mathcal{R}_{F_{p}}(p-1, m)$ is solved. 


\section{Computations}

We tested our results using the formulae for the dimensions as given in Section 2 with Magma [4]. For the dimension of the generalized Reed-Muller code we used the simplified form of Result 1 and the Magma function:

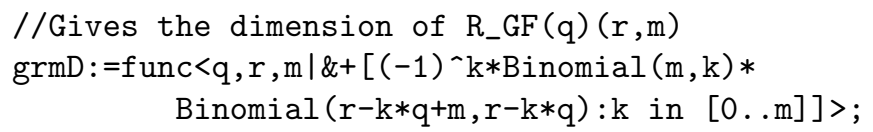

and for the dimension of the code spanned by the minimum-weight vectors we used Delsarte's Result 6 and the Magma function:

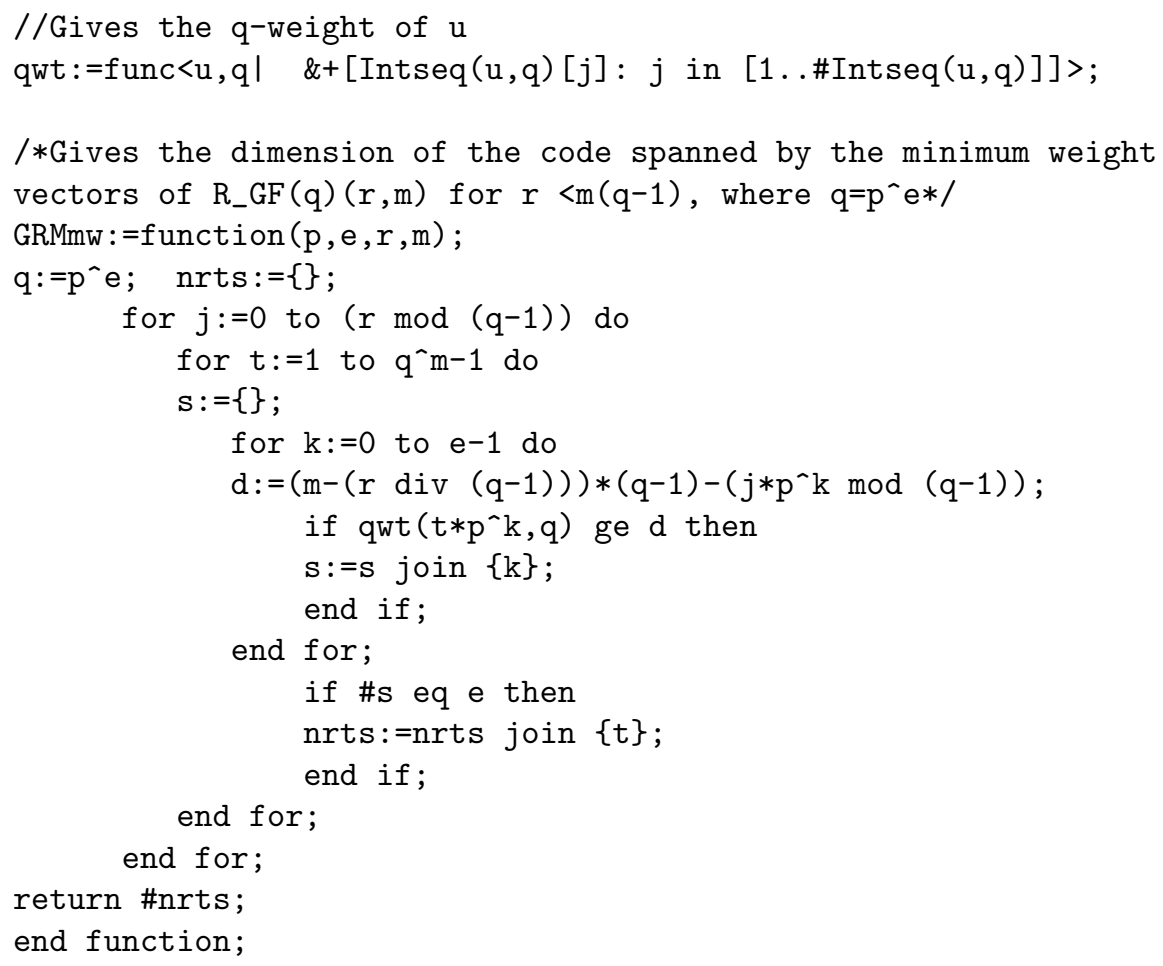

Some output using these Magma functions:

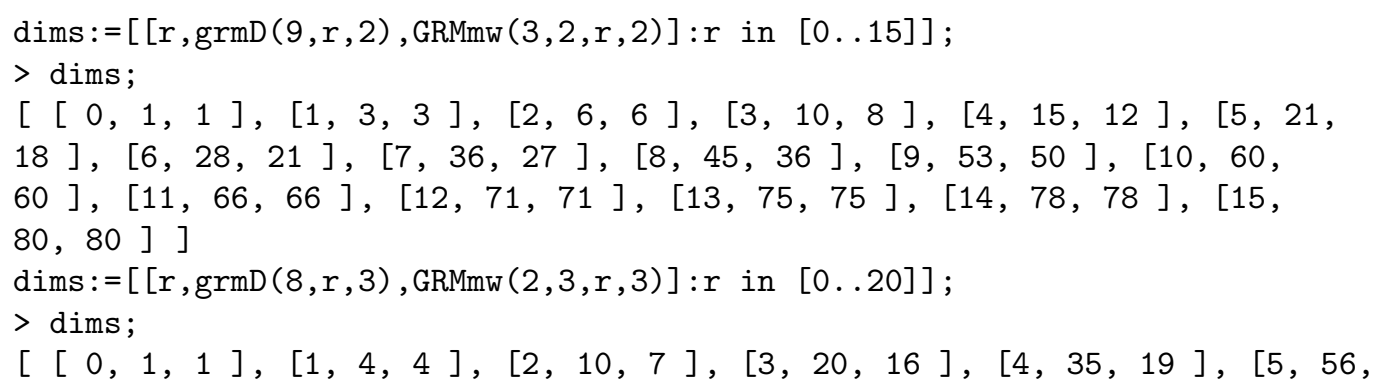


$28],[6,84,37],[7,120,64],[8,162,127],[9,208,172],[10$, $256,231],[11,304,258],[12,350,298],[13,392,328],[14,428$, $373],[15,456,443],[16,477,474],[17,492,492],[18,502,502$ ]$,[19,508,508],[20,511,511]]$

Notice that the values of $r$ for which the dimensions become the same agrees with our theorem.

\section{Acknowledgement}

The authors thank the reviewers for their careful reading and constructive comments.

\section{References}

[1] E. F. Assmus, Jr. and J. D. Key. Designs and their Codes. Cambridge: Cambridge University Press, 1992. Cambridge Tracts in Mathematics, Vol. 103 (Second printing with corrections, 1993).

[2] E. F. Assmus, Jr. and J. D. Key. Polynomial codes and finite geometries. In V. S. Pless and W. C. Huffman, editors, Handbook of Coding Theory, pages 1269-1343. Amsterdam: Elsevier, 1998. Volume 2, Part 2, Chapter 16.

[3] Thierry Berger and Pascale Charpin. The automorphism group of Generalized Reed-Muller codes. Discrete Math., 117:1-17, 1993.

[4] Wieb Bosma and John Cannon. Handbook of Magma Functions. Department of Mathematics, University of Sydney, November 1994.

[5] Pascale Charpin. Open problems on cyclic codes. In V. S. Pless and W. C. Huffman, editors, Handbook of Coding Theory, pages 963-1063. Amsterdam: Elsevier, 1998. Volume 1, Part 1, Chapter 11.

[6] P. Delsarte, J. M. Goethals, and F. J. MacWilliams. On generalized Reed-Muller codes and their relatives. Inform. and Control, 16:403-442, 1970.

[7] Philippe Delsarte. On cyclic codes that are invariant under the general linear group. IEEE Trans. Inform. Theory, 16:760-769, 1970.

[8] S. Gao and J. D. Key. Bases of minimum-weight vectors for codes from designs. Finite Fields Appl., 4:1-15, 1998.

[9] Brian Mortimer. Some problems on permutation groups: affine groups and modular permutation representations. $\mathrm{PhD}$ thesis, Westfield College, University of London, 1977. 